\title{
THE THREE-DIMENSIONAL QUASI-PERIODIC BOUNDARY ELEMENT METHOD: IMPLEMENTATION, EVALUATION, AND USE CASES
}

\author{
H. ZIEGELWANGER, P. REITER \& M. CONTER \\ AIT Austrian Institute of Technology $\mathrm{GmbH}$, Austria.
}

\begin{abstract}
The boundary element method (BEM) is a widely used engineering tool in acoustics. The major disadvantage of the three-dimensional boundary element method (3D-BEM) is its computational cost, which increases with the size of the simulated obstacle and the simulated wave number. Thus, the geometrical details of the obstacle and the simulated frequency range are limited by computer speed and memory.

The computational cost for simulating large obstacles like noise barriers is often reduced by applying the two-dimensional boundary element method (2D-BEM) on three-dimensional obstacles. However, the 2D-BEM limits the geometry of the boundary to obstacles with a one-dimensionally constant profile. An interesting compromise solution between the 2D-BEM and the 3D-BEM is the quasi-periodic boundary element method (QP-BEM). The QP-BEM allows the simulation of periodically repetitive complex three-dimensional structures and periodic sound fields while keeping the computational cost at a reasonable level.

In this study, first, the QP-BEM was implemented and coupled with the fast multipole method. Second, the QP-BEM was used to simulate the sound field radiated by a simple geometric object, i.e., a uniformly vibrating cylinder. Results were compared to an analytic solution, for the evaluation of the numerical accuracy of our QP-BEM implementation. For the demonstration of some use cases, third, the QP-BEM was used to simulate the sound field scattered by a sonic crystal noise barrier and a noisebarrier top element.

Keywords: acoustics, boundary element method, diffraction, fast multipole method, helmholtz equation, noise barriers, periodicity, scattering.
\end{abstract}

\section{INTRODUCTION}

The boundary element method (BEM) [1] is a widely used engineering tool to simulate the radiation, scattering, and diffraction of acoustic waves for a bounding geometry, i.e., the obstacle. Applications include the design of exhaust pipes, loudspeaker waveguides, virtual acoustics as well as noise barriers [2]. For instance, the BEM was used to investigate the acoustic properties of various noise-barrier materials and noise-barrier shapes, e.g., the general noise-barrier shape [3] or the design of noise-barrier top edges [4].

The major disadvantage of the three-dimensional boundary element method (3D-BEM) is its computational cost, i.e., the required amount of physical memory (RAM) and the computation time. The computational cost increases with the size of the obstacle and the simulated wave number. For instance, the calculation of the standardized sound diffraction index (defined in the European standard EN 16272-4) of a noise barrier's top edge stipulates the simulation of a $10 \mathrm{~m}$ long and $4 \mathrm{~m}$ high noise barrier in third-octave bands ranging from $100 \mathrm{~Hz}$ to $5,000 \mathrm{~Hz}$ resulting in a numerical problem of approximately two million unknowns (when considering eight constant rectangular elements per wavelength in the simulation [5]). However, the available amount of RAM limits the number of unknowns in the numerical calculation and CPU speed defines the computation time to solve the numerical problem.

In the field of acoustics, the computational cost for simulating large obstacles like noise barriers and high frequencies is often reduced by applying the two-dimensional boundary 
element method (2D-BEM) on three-dimensional obstacles [6]. However, the 2D-BEM limits the boundary geometry to obstacles with a one-dimensionally constant cross section. An interesting compromise solution between the 2D-BEM and the 3D-BEM is the quasi-periodic boundary element method (QP-BEM) [7]. The QP-BEM allows the simulation of periodically repetitive complex three-dimensional structures, e.g., noise barriers, while keeping the computational cost at a reasonable level. For instance, the calculation of the sound diffraction index of a periodic noise barrier with a period width of $5 \mathrm{~cm}$ can be reduced to a numerical problem of approximately 10,000 unknowns. However, as a consequence of the mathematical formulation of the QP-BEM also the sound field has to be periodic, e.g., produced by a periodic point source, or one-dimensionally constant, e.g., a plane wave or a cylindrical wave, in the simulation.

In this study, we present an implementation of the QP-BEM coupled with the fast multipole method (FMM). We start with the mathematical formulation of the BEM, the QP-BEM, and the FMM approximation. Our QP-BEM implementation was then used to simulate the sound field radiated by a simple geometric object, i.e., a vibrating cylinder, and the results were compared to an analytic solution to evaluate the numerical accuracy of the code. Finally, to demonstrate some use cases the QP-BEM was used to simulate the sound field scattered by a sonic crystal noise barrier and a noise-barrier top element.

\section{METHODS}

\subsection{Boundary element method}

Starting from the Helmholtz (see Figure 1) equation, the sound field in an exterior domain $\Omega_{\mathrm{e}}$ bounded by the surface $\Gamma$ of an object $\Omega$ is determined by the boundary integral equation (BIE):

$$
\begin{aligned}
& -\frac{1}{2} \phi(\mathbf{x})+\int_{\Gamma} H(\mathbf{x}, \mathbf{y}) \phi(\mathbf{y}) \mathrm{d} \mathbf{y} \\
& -\phi_{i n c}(\mathbf{x})+\int_{\Gamma} G(\mathbf{x}, \mathbf{y}) v(\mathbf{y}) \mathrm{d} \mathbf{y} \quad \mathbf{x} \in \Gamma,
\end{aligned}
$$

where $\phi(x)=-\frac{p(\boldsymbol{x})}{i \omega \rho}$ is the sound velocity potential, $p(\boldsymbol{x})$ is the sound pressure, and $v(\mathbf{x})$ is the particle velocity at point $\mathbf{x} . \rho$ is the density of the medium in $\Omega_{\mathrm{e}}$ (e.g. air), $\omega$ is the circular frequency and $\phi_{i n c}$ is the sound velocity potential of the incoming sound field. $G(\boldsymbol{x}, \boldsymbol{y})=\frac{e^{\mathrm{i} k|\boldsymbol{y}-\boldsymbol{x}|}}{4 \pi|\boldsymbol{y}-\boldsymbol{x}|}$ are the Green's function and its derivative with respect to the normal vector on $\Gamma \cdot k=\frac{\omega}{c}$ is the wave number, $c$ is the speed of sound and $\mathrm{i}$ is the imaginary unit. By the use of collocation with constant basis functions [8] and by discretizing the boundary as mesh, the BIE is transformed into:

$$
\begin{aligned}
& -\frac{1}{2} \phi\left(x_{i}\right)+\sum_{j} \phi_{j} \int_{\Gamma_{j}} H\left(x_{i}, y\right) \mathrm{d} \boldsymbol{y}= \\
& -\phi_{i n c}\left(\boldsymbol{x}_{i}\right)+\sum_{j} v_{j} \int_{\Gamma_{j}} G\left(\boldsymbol{x}_{i}, \boldsymbol{y}\right) \mathrm{d} \boldsymbol{y}, \quad \boldsymbol{x}_{i} \in \Gamma,
\end{aligned}
$$




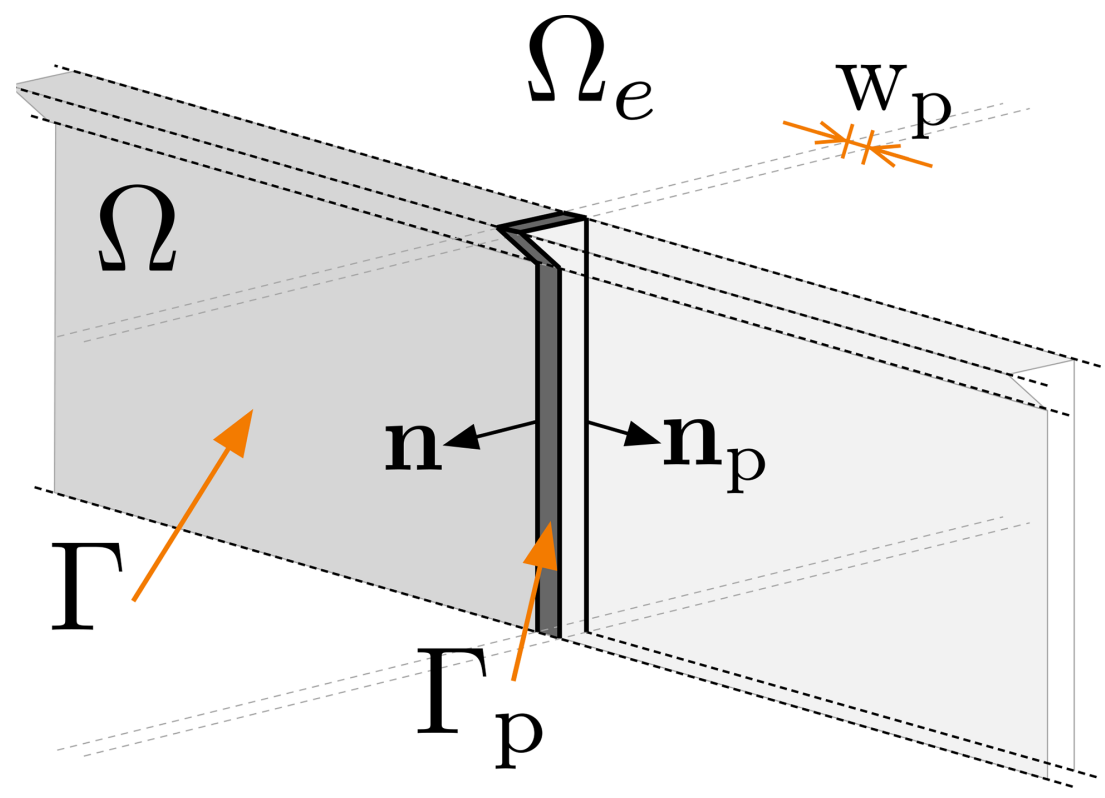

Figure 1: Sketch of the an infinitely long noise barrier to illustrate the acoustic problem of interest. $\Omega$ is the periodic obstacle, i.e., the noise barrier, $\Omega_{\mathrm{e}}$ the exterior domain, $\Gamma$ the boundary of $\Omega, \mathbf{n}$ the normal vector on $\Gamma, \Gamma_{\mathrm{p}}$ the boundary in a period of the obstacle, $\mathrm{w}_{\mathrm{p}}$ the width of a period, and $\mathbf{n}_{\mathrm{p}}$ the vector orthonormal to the cross section of the periodic obstacle.

where $j$ is the index of elements defining $\Omega$ and $i$ is the index of collocation nodes at the center of each element.

\subsection{Periodic BEM}

For periodic obstacles (e.g. noise barriers) and periodic or one-dimensionally constant sound fields, eqn 2 can be simplified to an infinite sum over periods of the object and sound field [7]:

$$
\begin{aligned}
& -\frac{1}{2} \phi\left(\boldsymbol{x}_{i}\right)+\sum_{j} \phi_{j} \sum_{\mathrm{p}=-\infty}^{\infty} \int_{\Gamma_{j}, \mathrm{p}} H\left(\boldsymbol{x}_{i}, \boldsymbol{y}+\mathrm{p} \boldsymbol{n}_{\mathrm{p}} w_{\mathrm{p}}\right) \mathrm{d} \boldsymbol{y}= \\
& -\phi_{i n c}\left(\boldsymbol{x}_{i}\right)+\sum_{j} v_{j} \sum_{\mathrm{p}=-\infty}^{\infty} \int_{\Gamma_{j}, \mathrm{p}} G\left(\boldsymbol{x}_{i}, \boldsymbol{y}+\mathrm{p} \boldsymbol{n}_{\mathrm{p}} w_{\mathrm{p}}\right) \mathrm{d} \boldsymbol{y}, \quad \boldsymbol{x}_{i} \in \Gamma,
\end{aligned}
$$

where $\mathrm{p}$ is the index of the period and $\mathbf{n}_{\mathrm{p}}$ is the orthonormal vector of the period cross section. $i$ and $j$ now represent the element and collocation node indexes in the periodic cut out $\Gamma_{\mathrm{p}}$ only.

A periodic sound field can be generated, for instance, by an infinite line of equidistant point sources:

$$
\phi_{i n c}\left(\boldsymbol{x}_{i}\right)=Q_{0} \sum_{\mathrm{p}=-\infty}^{\infty} \frac{e^{\mathrm{i} k\left|\boldsymbol{x}^{*}+\mathrm{p} n_{\mathrm{p}} w_{\mathrm{p}}-x_{i}\right|}}{4 \pi\left|\boldsymbol{x}^{*}+\mathrm{p} \boldsymbol{n}_{\mathrm{p}} w_{\mathrm{p}}-\boldsymbol{x}_{i}\right|}
$$


where $x^{*}$ represents the position of a point source in the period of the object and $Q_{0}$ is the source strength of a point source.

\subsection{Quasi-periodic BEM}

In the QP-BEM, the infinite sums in eqn 3 and eqn 4 are truncated after $P$ periods:

$$
\begin{aligned}
& -\frac{1}{2} \phi\left(\boldsymbol{x}_{i}\right)+\sum_{j} \phi_{j} \sum_{\mathrm{p}=-P}^{P} \int_{\Gamma_{j}, \mathrm{p}} H\left(\boldsymbol{x}_{i}, \boldsymbol{y}+\mathrm{p} \boldsymbol{n}_{\mathrm{p}} w_{\mathrm{p}}\right) \mathrm{d} \boldsymbol{y}= \\
& -\phi_{i n c}\left(\boldsymbol{x}_{i}\right)+\sum_{j} v_{j} \sum_{\mathrm{p}=-P}^{P} \int_{\Gamma_{j}, \mathrm{p}} G\left(\boldsymbol{x}_{i}, \boldsymbol{y}+\mathrm{p} \boldsymbol{n}_{\mathrm{p}} w_{\mathrm{p}}\right) \mathrm{d} \boldsymbol{y}, \quad \boldsymbol{x}_{i} \in \Gamma .
\end{aligned}
$$

For the periodic object and sound field, the Kirchoff-Helmholtz integral is then used to calculate the sound field in the exterior domain:

$$
\begin{aligned}
\phi(\boldsymbol{x}) \approx & \sum_{j} \phi_{j} \sum_{\mathrm{p}=-P}^{P} \int_{\Gamma_{j}, \mathrm{p}} H\left(\boldsymbol{x}_{i}, \boldsymbol{y}+\mathrm{p} \boldsymbol{n}_{\mathrm{p}} w_{\mathrm{p}}\right) \mathrm{d} \boldsymbol{y}-\phi_{i n c}(\boldsymbol{x})+ \\
& \sum_{j} v_{j} \sum_{\mathrm{p}=-P}^{P} \int_{\Gamma_{j}, \mathrm{p}} G\left(\boldsymbol{x}, \boldsymbol{y}+\mathrm{p} \boldsymbol{n}_{\mathrm{p}} w_{\mathrm{p}}\right) \mathrm{d} \boldsymbol{y}, \quad \boldsymbol{x} \in \Omega_{e} .
\end{aligned}
$$

2.4 Fast multipole formulation

In the fast multipole method, the boundary mesh is clustered and the Green's function is approximated for far-field clusters by:

$$
G(\boldsymbol{x}, \boldsymbol{y}) \approx \frac{\mathrm{i} k}{4 \pi} \int_{S} e^{\mathrm{i} k s\left(\boldsymbol{x}-z_{2}\right)} M_{L}(s, D) e^{i k s\left(\boldsymbol{y}-z_{1}\right)},
$$

where

$$
M_{L}(s, D):=\sum_{l=0}^{L} \sum_{\mathrm{p}=-P}^{P}(2 l+1) i^{l} h_{l}^{(1)}\left(k\left\|D+\mathrm{p} \boldsymbol{n}_{\mathrm{p}} w_{\mathrm{p}}\right\|\right) P_{l}\left(s \frac{D+\mathrm{p} \boldsymbol{n}_{\mathrm{p}} w_{\mathrm{p}}}{\left\|D+\mathrm{p} \boldsymbol{n}_{\mathrm{p}} w_{\mathrm{p}}\right\|}\right)
$$

$z_{1}$ and $z_{2}$ are the center points of two clusters, $\mathrm{D}=z_{2}-z_{1}, \mathrm{~S}$ is the unit sphere surface, $h_{l}^{(1)}$ denotes the spherical Hankel function of the first kind and order $l, P_{l}$ are $l$-th order Legendre polynomials, and $L$ is the multipole truncation parameter.

Then, the system matrix in the QP-BEM can be separated into a near-field and a far-field component:

$$
\left(\sum_{\mathrm{p}} N_{\mathrm{p}}+S \sum_{\mathrm{p}} D_{\mathrm{p}} T\right) \phi=b,
$$

where $N_{\mathrm{p}}$ is the sparse near-field matrix, $T$ the far-field signature, $D_{\mathrm{p}}$ the translation matrix and $S$ the near-field signature. The right-hand-side $b$ is defined by the incident field and boundary conditions. For more details about the fast multipole method refer to $[9,10]$.

\subsection{Implementation}

The QP-BEM was implemented based on an existing open-source BEM code (the BEMSolver from Mesh2HRTF (Version 0.1.2) was used [10, 11], available from http://mesh2hrtf. 
sourceforge.net). To ensure a unique solution in the calculations, the Burton-Miller approach was used [12]. All simulations ran on a Linux desktop PC, equipped with two Intel Xeon E5-2667 processors $(3.30 \mathrm{GHz})$ and $128 \mathrm{~GB}$ RAM.

\section{EVALUATION}

For the evaluation of the implemented QP-BEM, the sound field of an infinitely long and uniformly vibrating cylinder was simulated and compared to an analytic solution. The radius $R$ of the cylinder was $20 \mathrm{~cm}$. In all calculations, the sound pressure on the boundary and in the exterior domain was evaluated for frequencies ranging from $100 \mathrm{~Hz}$ to $5,000 \mathrm{~Hz}$.

For the analytic solution, the sound field of an infinitely long and uniformly vibrating cylinder was calculated by [13]:

$$
p_{r e f}(\boldsymbol{x})=\mathrm{i} \rho c \frac{H_{0}^{(2)}(k|\boldsymbol{x}|)}{H_{1}^{(2)}(k R)} v_{0}
$$

where $H_{0}^{(2)}$ and $H_{1}^{(2)}$ are Hankel functions and $v_{0}$ is the orthogonal particle velocity at the surface of the cylinder. For the QP-BEM calculation, a cut out of the infinitely long cylinder was modeled as a mesh. The period width $w_{p}$ was defined to be $40 \mathrm{~cm}$ and the mesh consisted of 20,480 rectangular elements. The edge length was $5 \mathrm{~mm}$ everywhere, which corresponded to approximately 14 elements per wave length at $5 \mathrm{kHz}$.

For the quantification of the accuracy of the QP-BEM implementation, the relative numerical error for field points lying on the boundary surface $\Gamma$ and for field points in the exterior domain $\Omega_{\mathrm{e}}$ was evaluated:

$$
\begin{aligned}
& e_{2}^{\Gamma}=\frac{\left\|p_{\text {num }}^{\Gamma}-p_{\text {ref }}^{\Gamma}\right\|_{2}}{\left\|p_{\text {ref }}^{\Gamma}\right\|_{2}}, \quad e_{2}^{\Omega_{e}}=\frac{\left\|p_{\text {num }}^{\Omega_{e}}-p_{\text {ref }}^{\Omega_{e}}\right\|_{2}}{\left\|p_{\text {ref }}^{\Omega_{e}}\right\|_{2}} \\
& e_{\infty}^{\Gamma}=\frac{\left\|p_{\text {num }}^{\Gamma}-p_{\text {ref }}^{\Gamma}\right\|_{\infty}}{\left\|p_{\text {ref }}^{\Gamma}\right\|_{\infty}}, \quad e_{\infty}^{\Omega_{e}}=\frac{\left\|p_{\text {num }}^{\Omega_{e}}-p_{\text {ref }}^{\Omega_{e}}\right\|_{\infty}}{\left\|p_{\text {ref }}^{\Omega_{e}}\right\|_{\infty}},
\end{aligned}
$$

where $\|\cdot\|_{2}$ and $\|\cdot\|_{\infty}$ denote the Euclidean and infinity norm for discrete values of the sound pressure, respectively.

Figures 2 and 3 show the relative numerical error on $\Gamma$ and in $\Omega_{\mathrm{e}}$ based on the Euclidean and infinity norm, respectively, for different truncation parameters ranging from $P=0$ to $P=1,000$. For $P>300$ (corresponding to a cylinder length of $240.4 \mathrm{~m}$ used in the QP-BEM calculations), the relative numerical error decreased below $0.5 \%$.

\section{USE CASES}

Relevant use cases of the QP-BEM are noise barrier simulations, e.g., numerical calculation of the insertion loss or diffraction index. Thus, we show two use cases of noise barrier simulations. In the first one, the sound diffraction at a noise barrier's top edge consisting of quarter-wave resonators was simulated. In the second one, the sound field inside and behind a sonic crystal noise barrier consisting of finite-length vertical cylinders was simulated. Note that both use cases are not computable by the 2D-BEM or the 3D-BEM. On the one hand, it is not possible to represent sonic crystals of finite length or arbitrarily shaped quarter-wave resonators in the 2D-BEM. On the other hand, RAM requirements for simulating those examples by the 3D-BEM exceed the specification of current desktop PCs, even when coupling the BEM with the FMM. 
(a) $f$ in $k H z$

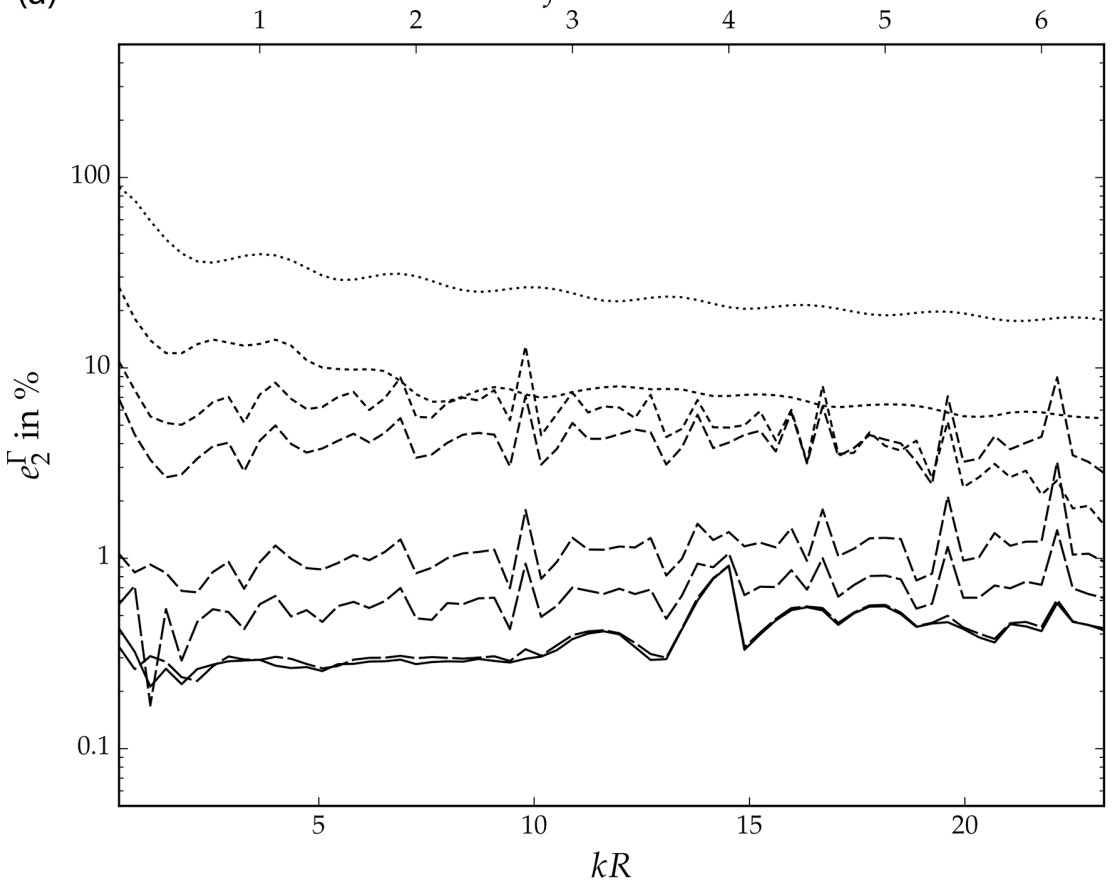

(b)

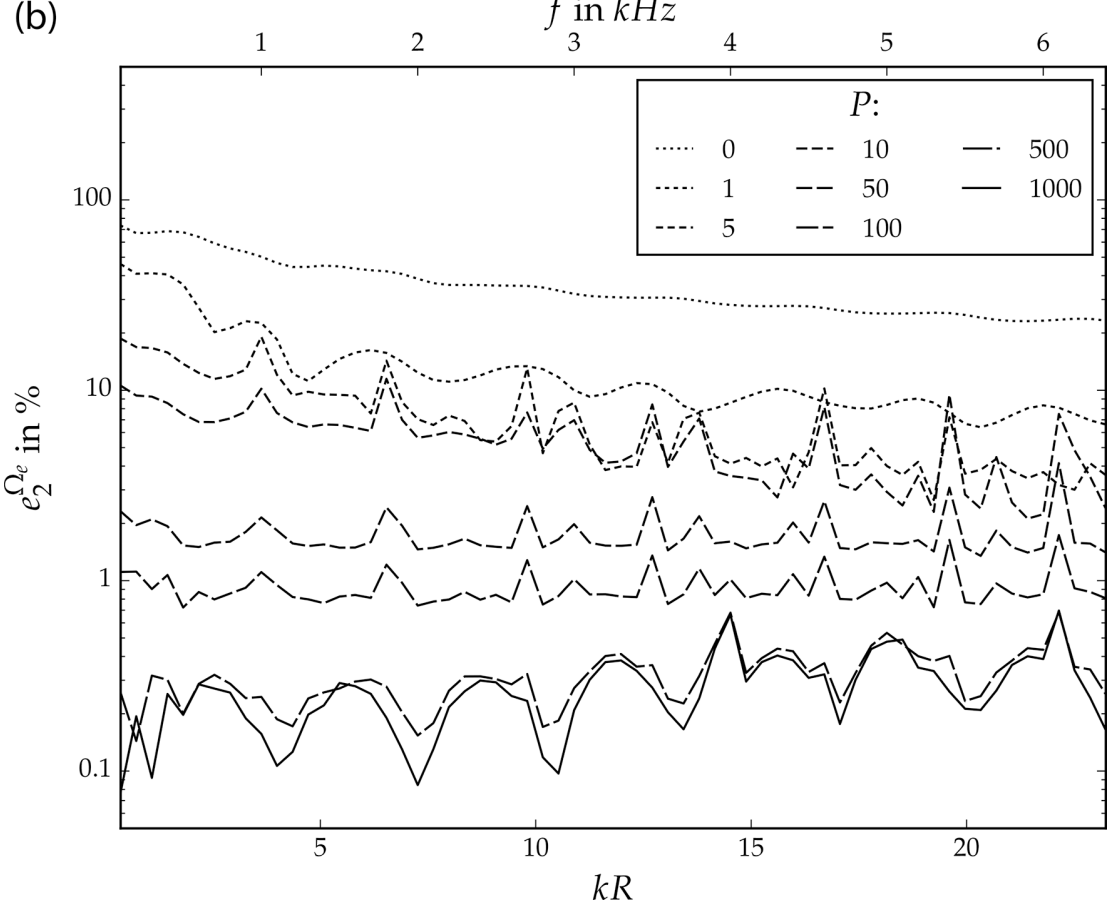

Figure 2: Relative numerical error for the Euclidean norm. (a) Relative numerical error on the boundary. (b) Relative numerical error in the exterior domain. 

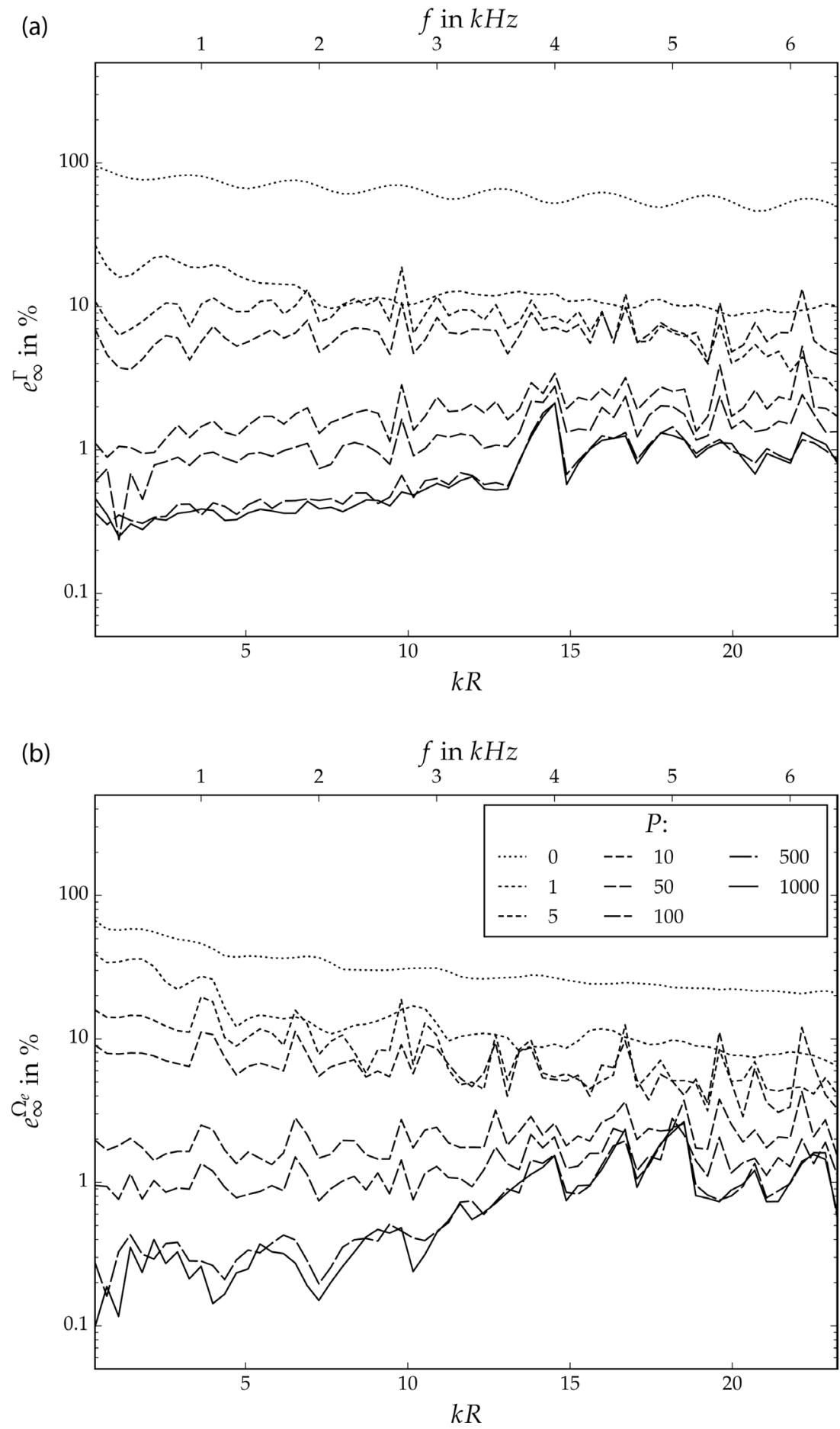

Figure 3: Relative numerical error for the infinity norm. (a) Relative numerical error on the boundary; (b) Relative numerical error in the exterior domain. 


\subsection{Noise barrier top element}

When developing a noise barrier, the design of its top edge is of special interest since the top edge is mainly responsible for the diffraction. As an example, quarter-wave resonators can be used to damp the diffracted sound at the top edge. The QP-BEM was used to simulate the diffraction at a noise barrier's top edge consisting of quarter-wave resonators. The QP-BEM reduced the numerical problem to the period width of the periodic noise barrier. The quarter-wave resonators and the mesh of one period of the noise barrier are shown in Fig. 4a.

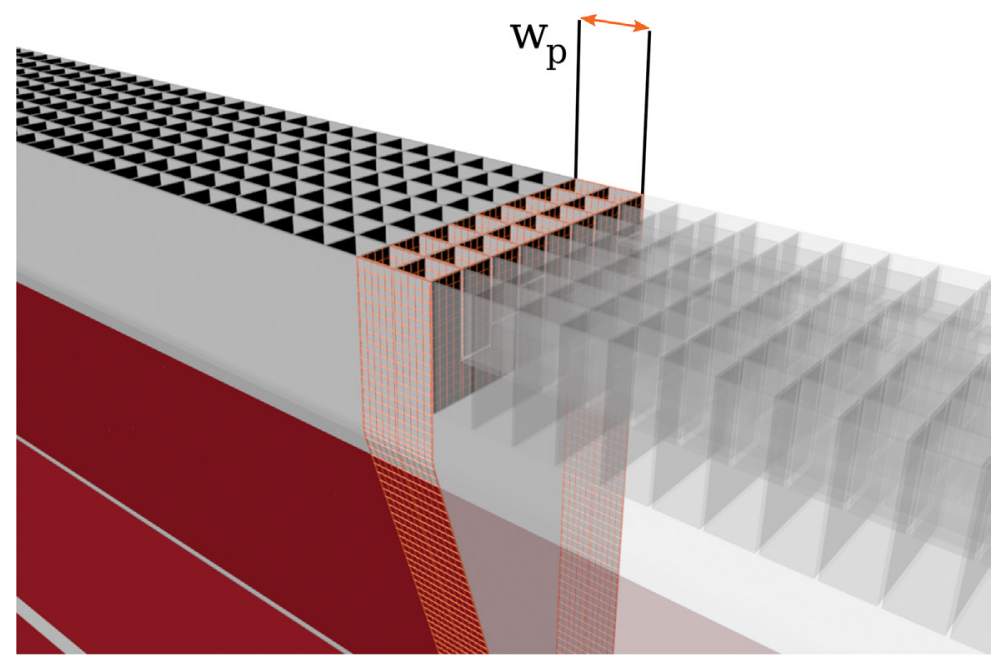

(a)

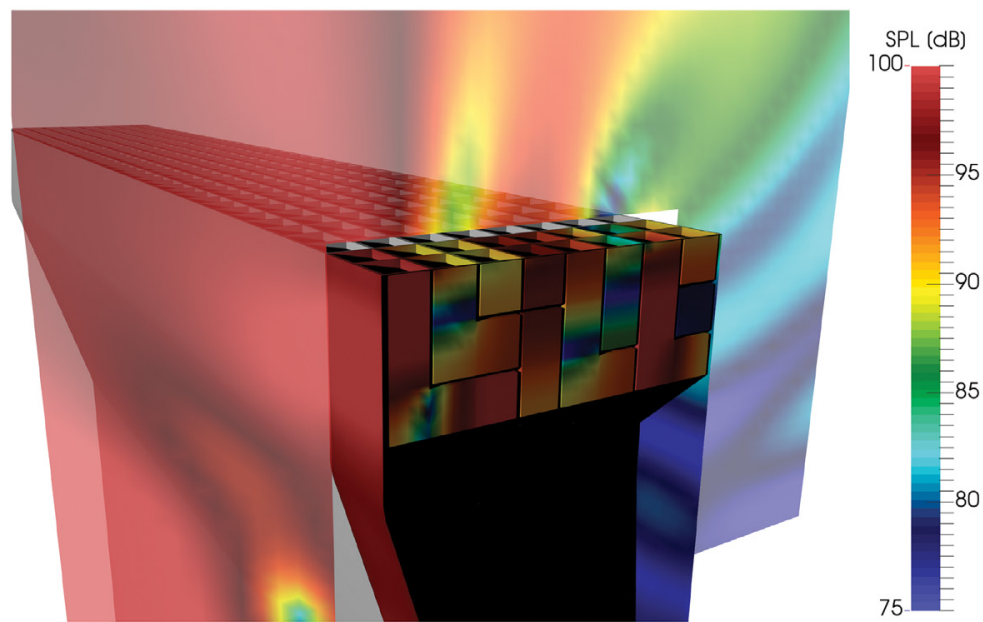

(b)

Figure 4: Simulation result of the sound diffraction at a noise barrier's top edge $(1,096 \mathrm{~Hz})$. (a) 3D-view of a noise barrier top edge consisting of acoustic resonators which should damp the sound diffraction. The orange grid represents the mesh used for the QP-BEM simulation. (b) Sound field simulated with the QP-BEM around the top edge of the noise barrier and on the surface of the acoustic resonators. 
The period width $\mathrm{w}_{\mathrm{p}}$ was $9 \mathrm{~cm}$, the mesh consisted of 17,992 rectangular elements and the average edge length in the mesh was approximately $1 \mathrm{~cm}$. For the simulation, a periodic point source was placed in front of the top edge with a distance of $2 \mathrm{~m}$. The truncation parameter $P$ was set to 1,500 , which approximately matches the length used in the cylinder example $(P=300$, compare section 3). The calculated sound pressure is shown in Fig. 4b. The sound

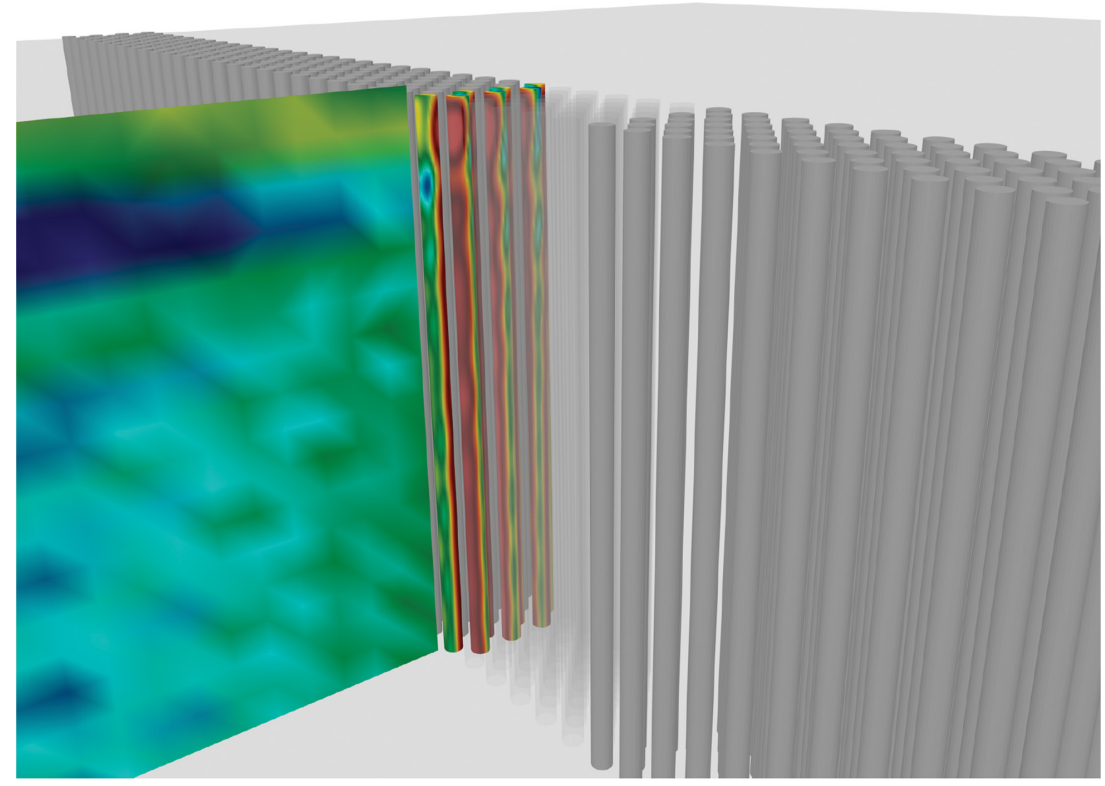

(a)

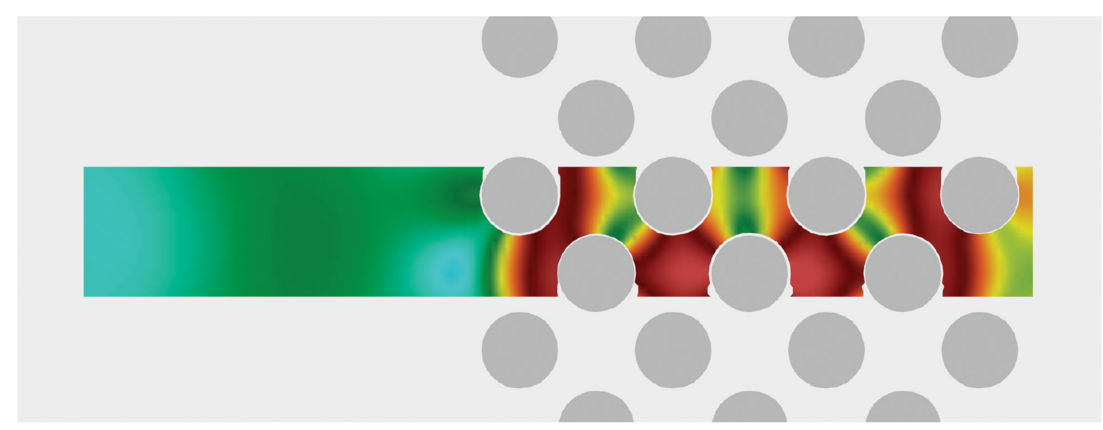

(b)

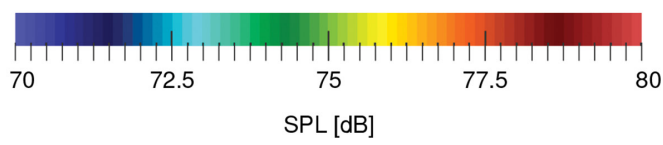

Figure 5: Sound field simulated for a sonic crystal noise barrier at $816 \mathrm{~Hz}$. (a) $806 \mathrm{~Hz}$, 3-D view; (b) $806 \mathrm{~Hz}$, top view. 
pressure vanishes at different locations in the resonators (corresponding to the simulated frequency and the length of the resonators), e.g., the sound pressure vanishes at the entrance of the resonators of the third resonator row.

\subsection{Sonic crystal noise barrier}

A sonic crystal noise barrier consists of equidistantly placed rigid cylinders. The radius of the cylinders and the distance between cylinders define its band gap frequency. The radius of the cylinders was $50 \mathrm{~mm}$, the cylinders were $3 \mathrm{~m}$ high, and the distance between the cylinders was $150 \mathrm{~mm}$. In the simulation, one period of the sonic crystal noise barrier consisted of two cylinder rows (compare Fig. 5b), the $\mathrm{w}_{\mathrm{p}}$ was $21.5 \mathrm{~cm}$, the mesh consisted of 38,514 rectangular elements, and the average edge length in the mesh was $1.5 \mathrm{~cm}$. The truncation parameter was set to $P=600$, which approximately matches the length used in the evaluation example $(P=300$, compare section 3$)$. A periodic point source was placed at ground level with a distance of $3 \mathrm{~m}$ to the noise barrier. The sound field in and behind the sonic crystal noise barrier simulated with the QP-BEM is shown in Figure 5.

\section{CONCLUSIONS}

The QP-BEM allows the simulation of periodic sound fields scattered at periodic 3D obstacles. The QP-BEM was implemented, coupled with the FMM and evaluated for a vibrating cylinder. The accuracy of the simulation increases, i.e., the relative numerical error decreases, for an increasing truncation parameter. In our implementation, the relative numerical error decreased below 0.5 percent when including more than 300 periods of the obstacle in the calculations. The QP-BEM can be used to simulate the sound field scattered around noise barriers with periodic geometry, which cannot be simulated by the 2D-BEM at all or by the 3D-BEM on current desktop PCs. Two possible use cases of the QP-BEM were shown, the sound field in a sonic crystal noise barrier and around a noise barrier top edge were simulated. In a following study, the QP-BEM will be used to optimize the shape of various noise barrier designs.

\section{REFERENCES}

[1] Brebbia, C. \& Dominguez, J., Boundary element methods for potential problems. Applied Mathematical Modelling, 1(7), pp. 372-378, 1977. https://doi.org/10.1016/0307-904X(77)90046-4

[2] Kurze, U.J., Noise reduction by barriers. The Journal of the Acoustical Society of America, 55(3), pp. 504-518, 1974. https://doi.org/10.1121/1.1914528

[3] Kasess, C.H., Kreuzer, W. \& Waubke, H., Deriving correction functions to model the efficiency of noise barriers with complex shapes using boundary element simulations. Applied Acoustics, 102, pp. 88-99, 2016. https://doi.org/10.1016/j.apacoust.2015.09.009

[4] Oldham, D.J. \& Egan, C.A., A parametric investigation of the performance of multiple edge highway noise barriers and proposals for design guidance. Applied Acoustics, 96, pp. 139-152, 2015. https://doi.org/10.1016/j.apacoust.2015.03.012

[5] Marburg, S., Six boundary elements per wavelength: Is that enough? Journal of Computational Acoustics, 10(1), pp. 25-51, 2002. https://doi.org/10.1142/S0218396X02001401 
[6] Duhamel, D., Efficient calculation of the 3D sound pressure field around a noise barrier. Journal of Sound and Vibration, 197(5), pp. 547-571, 1996. https://doi.org/10.1006/jsvi.1996.0548

[7] Fard, S., Peters, H., Kessissoglou, N. \& Marburg, S., Three-dimensional analysis of a noise barrier using a quasi-periodic boundary element method. The Journal of the Acoustical Society of America, 137(6), pp. 3107-3114, 2015. https://doi.org/10.1121/1.4921266

[8] Hunter, P. \& Pullan, A., 2002. FEM/BEM Notes.

[9] Chen, Z.S., Waubke, H. \& Kreuzer, W., A formulation of the fast multi-pole boundary element method for acoustic radiation and scattering from 3D structures. Journal of Computational Acoustics, 16(02), pp. 303-320, 2008. https://doi.org/10.1142/S0218396X08003725

[10] Ziegelwanger, H., Majdak, P. \& Kreuzer, W., Mesh2hrtf: Open-source software package for the numerical calculation of head-related transfer functions. Proceedings of the 22nd ICSV, Florence, IT, 2015.

[11] Ziegelwanger, H., Majdak, P. \& Kreuzer, W., Numerical calculation of listener-specific head-related transfer functions and sound localization. The Journal of the Acoustical Society of America, 138(1), pp. 208-222, 2015. https://doi.org/10.1121/1.4922518

[12] Burton, A.J. \& Miller, G.F., The application of integral equation methods to the numerical solution of some exterior boundary-value problems. Proceedings of the Royal Society of London a Mathematical and Physical Sciences, 323(1553), pp. 201-210, 1971. https://doi.org/10.1098/rspa.1971.0097

[13] Beranek, L.L. \& Mellow, T.J., Acoustics: Sound Fields and Transducers, Academic Press: Amsterdam, 2012. 